\title{
Estratégias de reabilitação fisioterapêutica em pacientes com neuropatia diabética: uma revisão sistemática
}

\author{
Daniela Xavier \\ Acadêmica do Curso de Bacharel em Fisioterapia Centro Universitário do Desenvolvimento \\ do Centro-Oeste (UNIDESC). Luziânia, Goiás, Brasil \\ $\triangle$ danielasantos3511@gmail.com \\ Thais Umbelino \\ Acadêmica do Curso de Bacharel em Fisioterapia Centro Universitário do Desenvolvimento \\ do Centro-Oeste (UNIDESC). Luziânia, Goiás, Brasil \\ $\triangle$ thais.umbelino@hotmail.com \\ Arielly Alves \\ Acadêmica do Curso de Bacharel em Fisioterapia Centro Universitário do Desenvolvimento \\ do Centro-Oeste (UNIDESC). Luziânia, Goiás, Brasil \\ $\triangle$ ariellyab2015@hotmail.com

\section{Ludmila Rocha Lemos} \\ Mestra em Engenharia Biomédica \\ Universidade de Brasília (UNB). Distrito Federal, Brasil \\ $\triangle \underline{\text { ludmilarochalemos@gmail.com }}$

\section{Leonardo Moreira Rabelo} \\ Acadêmico do Curso de Bacharel em Enfermagem Centro Universitário do Desenvolvimento \\ do Centro-Oeste (UNIDESC). Luziânia, Goiás, Brasil \\ $\bowtie$ leomrstark@gmail.com

\section{Krislayne Veras Alexandre} \\ Acadêmica do Curso de Bacharel em Enfermagem Centro Universitário do Desenvolvimento \\ do Centro-Oeste (UNIDESC). Luziânia, Goiás, Brasil \\ $\triangle$ krislaynev16@gmail.com

\section{Gabriela Meira de Moura Rodrigues} \\ Mestra em Engenharia Biomédica \\ Universidade de Brasília (UNB). Distrito Federal, Brasil \\ $\bowtie$ professoragabymeira@gmail.com
}

\section{Resumo:}

Introdução: O Diabetes Mellitus (DM) é uma doença crônica caracterizada pela elevação da glicose no sangue acima da taxa normal. Os pacientes com DM podem manifestar baixa sensibilidade, irregularidade na propriocepção, baixa flexibilidade e atrofia muscular. Objetivo: Responder a seguinte pergunta: Quais as estratégias que podem ser utilizadas na reabilitação fisioterapêutica de pacientes com neuropatia diabética (ND)? Metodologia: Estudo realizado por meio de revisão sistemática. Foram utilizadas 27 pesquisas publicadas entre 2007 a 2019 para a composição desse 
artigo. Para o desenvolvimento dos resultados, foi realizado uma síntese minuciosa nos bancos de dados Google Acadêmico, PubMed, Scielo, Medline e Repositórios Universitários sobre as formas de tratamentos fisioterapêuticos da neuropatia diabética. Resultados: Previamente, foram selecionadas 24 pesquisas e destas usadas 5 para compor um quadro com as medidas que podem ser utilizadas na reabilitação fisioterapêutica em indivíduos com neuropatia diabética. Todos os 5 estudos conseguiram resultados positivos com os seus respectivos tratamentos. Discussão: Ainda é escasso na literatura formas de tratamento da neuropatia. Isso precisa ser mudado, pois como visto o tratamento traz benefícios aos acometidos por esse agravo. Conclusão: O fisioterapeuta, por meio de seus cuidados, contribui para uma redução significativa dos danos causados pela neuropatia diabética. Este profissional necessita está capacitado para poder identificar o tipo de neuropatia que acomete o indivíduo e decidir pelo tratamento que melhor se adeque ao paciente.

Palavras-chave: Fisioterapia, Diabetes Mellitus, Neuropatias Diabéticas, Condutas Terapêuticas.

\title{
Physical Therapy Rehabilitation Strategies in Patients with Diabetic Neuropathy: A Systematic Review
}

\begin{abstract}
:
Introduction: Diabetes Mellitus (DM) is a chronic disease characterized by elevated blood glucose above the normal rate. Patients with DM may manifest low sensitivity, irregularity in proprioception, low flexibility and muscle atrophy. Objective: To answer the following question: What strategies can be used in the physical therapy rehabilitation of patients with diabetic neuropathy (ND)? Methodology: Study conducted through systematic review. Twenty-seven studies published between 2007 and 2019 were used for the composition of this article. For the development of the results, a thorough synthesis was performed in the Google Scholar, PubMed, Scielo, Medline and University Repositories databases on the forms of physiotherapeutic treatments of diabetic neuropathy. Results: Previously, 24 studies were selected and from these 5 were used to compose a chart with the measures that can be used in physical therapy rehabilitation in individuals with diabetic neuropathy. All 5 studies achieved positive results with their respective treatments. Discussion: There are still few ways to treat neuropathy in the literature. This needs to be changed, because as seen the treatment brings benefits to those affected by this condition. Conclusion: The physiotherapist, through their care, contributes to a significant reduction of the damage caused by diabetic neuropathy. This professional needs to be able to identify the type of neuropathy that affects the individual and decide on the treatment that best suits the patient.
\end{abstract}

Keywords: Physical Therapy Specialty, Diabetes Mellitus, Diabetic Neuropathies, Therapeutic Approaches.

\section{Estrategias de rehabilitación de fisioterapia en pacientes con neuropatía diabética: una revisión sistemática}

\section{Resumen:}

Introducción: la Diabetes Mellitus (DM) es una enfermedad crónica caracterizada por una glucemia elevada por encima de la tasa normal. Los pacientes con DM pueden manifestar baja sensibilidad, irregularidad en la propiocepción, baja flexibilidad y atrofia muscular. objetivo: responder la siguiente pregunta: ¿Qué estrategias se pueden utilizar en la rehabilitación de fisioterapia de pacientes con neuropatía diabética (ND)? Metodología: Estudio realizado mediante revisión sistemática. Veintisiete estudios publicados entre 2007 y 2019 se utilizaron para la composición de este artículo. Para el desarrollo de los resultados, se realizó una síntesis exhaustiva en las bases de datos de Google Scholar, PubMed, Scielo, Medline y University Repositories sobre las formas de 
tratamientos fisioterapéuticos de la neuropatía diabética. Resultados: Anteriormente, se seleccionaron 24 estudios y de estos 5 se usaron para componer una tabla con las medidas que se pueden usar en la rehabilitación de fisioterapia en individuos con neuropatía diabética. Los 5 estudios lograron resultados positivos con sus respectivos tratamientos. Resultados: Anteriormente, seleccionamos 24 estudios y 5 de estos se usarían para componer una tabla de medidas que podrían usarse para la rehabilitación de la fisioterapia en individuos con neuropatía diabética. Los 5 estudios lograron resultados positivos con sus tratamientos. Discusión: todavía hay pocas formas de tratar la neuropatía en la literatura. Esto necesita ser cambiado, porque como se ve, el tratamiento brinda beneficios a los afectados por esta afección. Conclusión: el fisioterapeuta, a través de su cuidado, contribuye a una reducción significativa del daño causado por la neuropatía diabética. Este profesional necesita poder identificar el tipo de neuropatía que afecta al individuo y decidir el tratamiento que mejor se adapte al paciente.

Palabras clave: Fisioterapia, Diabetes Mellitus, Neuropatías Diabéticas, Conductas Terapéuticas.

\section{INTRODUÇÃO}

O Diabetes Mellitus (DM), classificada como uma Doenças Crônicas Não-Transmissíveis (DCNT), é um distúrbio metabólico caracterizado por hiperglicemia persistente, isto é, uma consequência da falta de produção de insulina ou de sua ação, ou em ambos os casos, gerando assim, complicações durante um extenso período. A hiperglicemia persistente está relacionada a alguns fatores, tais como: os problemas crônicos micro e macrovasculares, a elevação da morbidade, diminuição da qualidade de vida e aumento da taxa de mortalidade. Os causadores genéticos, biológicos e ambientais da DM, ainda não são conhecidos integralmente (PETERMANN et al., 2015; SOCIEDADE BRASILEIRA DE DIABETES, 2017).

O diabetes é classificado em tipo 1 ou 2 e em outras formas específicas. O tipo 1 é congênito, se manifestando no nascimento ou até os 20 anos de idade e normalmente está associado a uma predisposição genética. Essa forma de DM é caracterizada pela presença de anticorpos do próprio corpo do indivíduo. Estes destroem as células secretoras de insulina, as células $\beta$ pancreáticas, isto promove à deficiência total desse hormônio, transformando a pessoa em insulinodependente. Já no diabetes tipo 2, ocorre uma resistência à ação da insulina, além disso, o pâncreas sofre desgaste por meio do método compensatório de hiperfuncionamento, reduzindo assim, a produção de insulina. Na maioria dos casos, este tipo aparece após os 40 anos de idade e está relacionado a causas genéticas e ambientais, como o sedentarismo e a obesidade. 0 paciente portador de diabetes tipo 2 não será necessariamente insulinodependente, pois pacientes que conseguem alcançar níveis normais de glicose com uso de medicação hipoglicemiante oral, controlando a alimentação e praticando exercícios físicos, não precisam de insulina exógena (MENDES et al., 2019). 
No ano de 2014, o DM foi a causadora de pelo menos $11 \%$ de todas às despesas com a saúde de adultos no mundo, apresentando um valor aproximado de 612 milhões de dólares. Ocasionou ainda 4,9 milhões de óbitos no mundo nesse mesmo período. No Brasil, dentre as mortes, essa enfermidade representa 5,2\%. Ela acomete principalmente populações de riscos, como os idosos e os indivíduos com baixa renda e escolaridade, sendo responsável ainda por 9\% de todos os gastos hospitalares do sistema de saúde do país (ARAUJO FILHO et al., 2017). O Brasil ocupa a quarta posição, com 14,3 milhões de casos de DM, no ranking mundial entre os 10 países com mais ocorrências dessa doença. Estimativas mostram que em 2040 o número de casos chegue a 23,3 milhões (INTERNATIONAL DIABETES FEDERATION, 2015).

Os pacientes portadores do DM, podem apresentar sensibilidade diminuída, irregularidade na propriocepção, pouca flexibilidade, atrofia muscular, desenvolver pé diabético e possuem maiores chances de quedas devido a problemas de equilíbrio. A instabilidade, em indivíduos com DM tipo 2, está relacionada a disfunção ou diminuição da comunicação sensorial periférica nos pés e a falta de eficiência do sistema nervoso central (SNC) para integrar corretamente com as informações usadas no controle postural. Portanto, as limitações motoras causadas pelas deficiências musculoesqueléticas, atrofias muscular e o não funcionamento autonômico, levam a transformações na estabilização postural e da biomecânica da locomoção, por causa das alterações na propriocepção, equilíbrio e coordenação motora. Logo, existe um crescimento do risco de quedas, ulcerações nos pés e amputação dos MMII (SALSABILI et al., 2011) (OLIVEIRA; FERREIRA; COUTINHO, 2017).

Dentre as várias comorbidades relacionadas ao controle ineficaz da glicemia, é necessário destacar a neuropatia como a mais comum das complicações microvasculares do DM, com mais de $50 \%$ dos pacientes com diabetes tipo 2 exibindo provas da neuropatia. As neuropatias são caracterizadas pela eliminação progressiva de fibras nervosas do sistema nervoso autonômico e somático. A fisiopatologia desses agravos ainda não é bem compreendida. O que demonstra a necessidade de mais estudos sobre o tema (LADE et al., 2016).

A neuropatia diabética (ND), uma complicação do diabetes, é formada por um conjunto de diferentes manifestações clínicas ou subclínicas, que afetam o sistema nervoso periférico 
(SNP). Pode apresentar-se de diversas maneiras clínicas e com diferentes mecanismos fisiopatológicos. (NASCIMENTO; PUPE; CAVALCANTI, 2016) (NASCIMENTO et al., 2015).

Existem três tipos de neuropatia diabética, são elas: neuropatia sensitiva, causadora de perda progressiva da percepção de pressão, temperatura, propriocepção e sensação de dor; neuropatia motora, que causa atrofiamento e enfraquecimento dos músculos intrínsecos do pé, originando deformidades e anormalidades no padrão da marcha e por último a neuropatia autonômica, nesta ocorre pouca ou nenhuma secreção sudorípara, resultando em ressecamento da derme com aparecimento de rachaduras e fissuras (BORGES; CARDOSO, 2010).

Aproximadamente $20 \%$ dos pacientes com ND apresentam dor neuropática, o que resulta em diminuição significativa da qualidade de vida e da capacidade funcional da pessoa. Vai ser também um fator de risco importante para o surgimento de úlceras e para o desenvolvimento de outras complicações microvasculares, além de deformidades e amputações dos membros inferiores (MMII). Esse agravo é o causador ainda do aumento dos números de internações hospitalares e mortalidade cardiovascular em clientes diabéticos. No ano de 2003, a estimativa do custo relacionado diretamente à ND e os seus problemas chega a U\$10,9 bilhões (NASCIMENTO; PUPE; CAVALCANTI, 2016).

Nesse contexto, a fisioterapia pesquisa e realiza o tratamento por meio de diversos procedimentos e ferramentas terapêuticas adequadas, promovendo assim, melhorias funcionais. Dessa forma, o objetivo do presente estudo é responder a seguinte pergunta: quais as estratégias que podem ser utilizadas na reabilitação fisioterapêutica de paciente com neuropatia diabética (ND)?

\section{MATERIAL E MÉTODOS}

O presente artigo foi elaborado a partir da utilização das seguintes etapas: (1) elaboração da pergunta de pesquisa; (2) procura na literatura; (3) escolha das fontes; (4) extração dos dados; (5) julgamento da qualidade; (6) resumo dos dados; (7) avaliação da qualidade das evidências e (8) redação dos resultados (GALVÃo; PEREIRA, 2014). 
A metodologia utilizada para a realização do artigo foi a de Revisão Sistemática. Este método é caracterizado por ser um resumo de diversas pesquisas desenvolvidas. Possui o objetivo de apresentar o melhor conhecimento possível sobre um determinado assunto específico, além de responder uma determinada pergunta específica (ROTHER, 2007; VIEIRA; HOSSN, 2015).

Para o desenvolvimento desse estudo foram utilizadas 27 pesquisas publicadas entre 2007 a 2019. Para a realização dos resultados dessa revisão foi feita uma busca, utilizando-se as palavras "fisioterapia", "neuropatia diabética", "tratamento" e "fisioterapêutica", por teses, anais e artigos pulicados entre 2000 e 2019 nos bancos de dados Google Acadêmico, PubMed, Scielo, Medline e Repositórios Universitários.

\section{RESULTADOS}

Considerando o foco e o objetivo geral desse estudo, foi pesquisado na literatura mundial tratamentos relacionados a neuropatia diabética. Os dados obtidos foram distribuídos em um quadro para melhor organização, além de haver uma breve síntese com o objetivo de ajudar os profissionais e pesquisadores que utilizarão qualquer uma dessas ferramentas.

Quadro 1 - Ações e estratégicas que podem ser usadas no tratamento da neuropatia diabética.

\begin{tabular}{|c|c|c|c|}
\hline AUTORES & TÍTULO & ANO & INTERVENÇÕES TERAPÊUTICAS \\
\hline $\begin{array}{l}\text { GOMES et } \\
\text { al., }\end{array}$ & $\begin{array}{l}\text { Efeitos da } \\
\text { intervenção } \\
\text { fisioterapêutica nas } \\
\text { respostas sensoriais } \\
\text { e funcionais de } \\
\text { diabéticos } \\
\text { neuropatas. }\end{array}$ & 2007. & $\begin{array}{l}\text { - Uso de cinesioterapia. } \\
\text { - Utilização de } 10 \text { sessões de fisioterapia: } \\
\text { - } 1^{\mathrm{a}} \text { à } 3^{\mathrm{a}} \text { sessão: alongamento de isquiotibiais, } \\
\text { tríceps sural e tibial anterior; mobilização } \\
\text { passiva de MMII até a Amplitude de } \\
\text { Movimento máxima (ADM) de cada indivíduo; } \\
\text { exercícios ativos resistidos isotônicos (com } \\
\text { os artelhos, pegar caneta e bolinha de }\end{array}$ \\
\hline
\end{tabular}




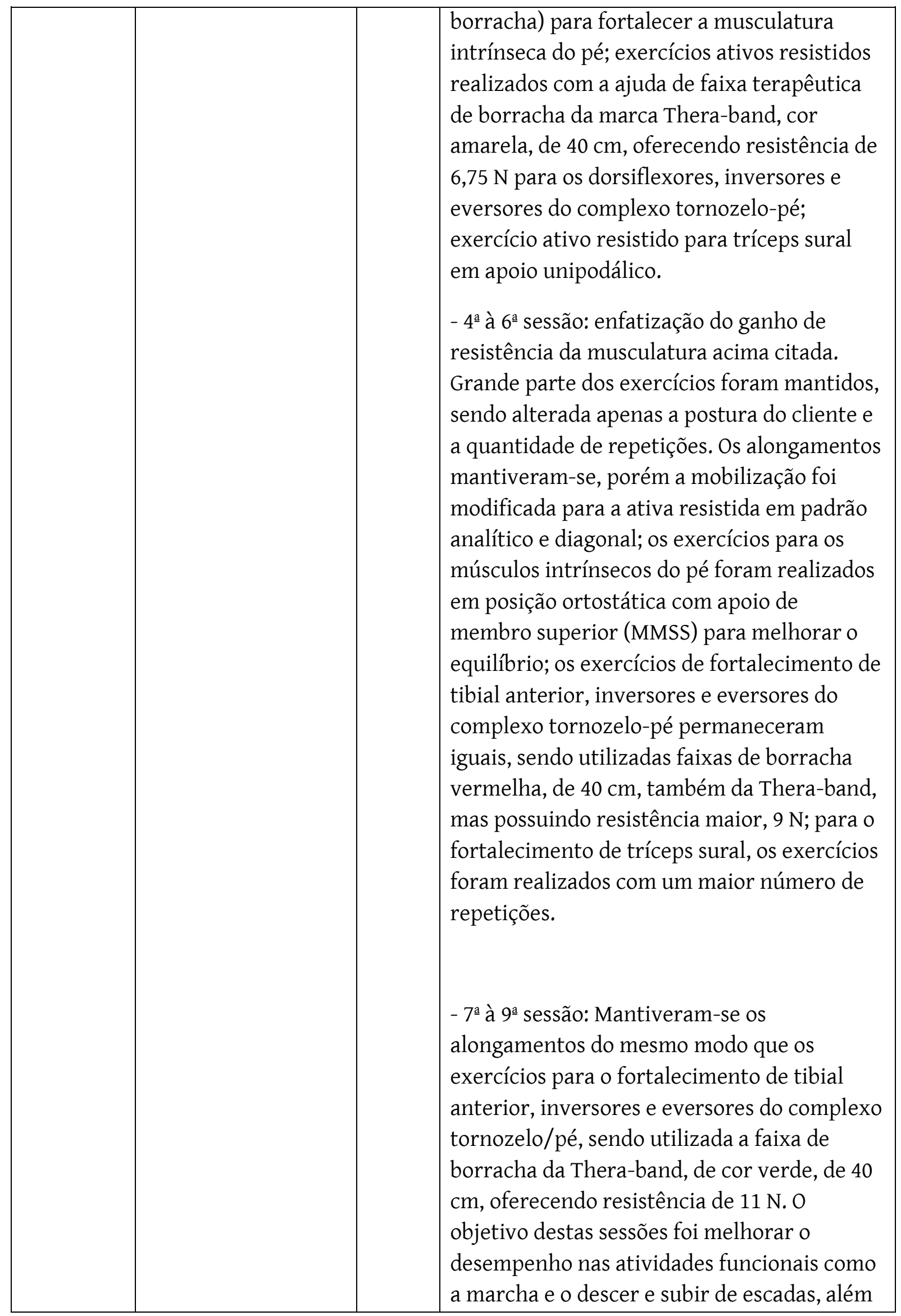




\begin{tabular}{|c|c|c|c|}
\hline & & & $\begin{array}{l}\text { da conquista de equilíbrio no decorrer da } \\
\text { postura estática e dinâmica. } \\
\text { - } 10^{\underline{a}} \text { sessão: Realizações de atividades } \\
\text { funcionais e término das orientações sobre o } \\
\text { autocuidado com os pés. }\end{array}$ \\
\hline $\begin{array}{l}\text { BARROS et } \\
\text { al., }\end{array}$ & $\begin{array}{l}\text { Impacto de } \\
\text { intervenção } \\
\text { fisioterapêutica na } \\
\text { prevenção do pé } \\
\text { diabético. }\end{array}$ & 2012. & $\begin{array}{l}\text { - Tratamento fisioterapêutico durante } 60 \\
\text { minutos; } \\
\text { - } 10 \text { minutos para os exercícios gerais de } \\
\text { alongamento; } \\
\text { - } 10 \text { minutos para as caminhadas; } \\
\text { - } 10 \text { minutos para as atividades do tipo ativo } \\
\text { livre e ativo resistido para a realização de } \\
\text { flexão plantar, dorsiflexão, inversão e } \\
\text { eversão do tornozelo; } \\
\text { - } 10 \text { minutos de exercícios de flexão, } \\
\text { extensão, abdução e adução dos dedos dos } \\
\text { membros inferiores; } \\
\text { - } 10 \text { minutos de exercícios para a } \\
\text { propriocepção dos pés, usando materiais } \\
\text { como areia e grãos; } \\
\text { - } 10 \text { minutos de massoterapia superficial e } \\
\text { profunda para os pés. } \\
\text { - Foram utilizadas bolas, faixas elásticas e } \\
\text { bastões para as atividades ativas livres e } \\
\text { pesos de meio quilo para os exercícios } \\
\text { resistidos; }\end{array}$ \\
\hline $\begin{array}{l}\text { YOOSEFIN } \\
\text { EJAD et al., }\end{array}$ & $\begin{array}{l}\text { The effectiveness of } \\
\text { a single session of } \\
\text { Whole-Body } \\
\text { Vibration in } \\
\text { improving the } \\
\text { balance and the } \\
\text { strength in type } 2 \\
\text { diabetic patients } \\
\text { with mild to }\end{array}$ & 2014. & $\begin{array}{l}\text { - Utilizar sessões na plataforma vibratória, já } \\
\text { que esta melhora significativamente a força } \\
\text { muscular dos membros inferiores e melhora } \\
\text { o equilíbrio. }\end{array}$ \\
\hline
\end{tabular}




\begin{tabular}{|c|c|c|c|}
\hline & $\begin{array}{l}\text { moderate degree of } \\
\text { peripheral } \\
\text { neuropathy: a pilot } \\
\text { study. }\end{array}$ & & \\
\hline FURIERI. & $\begin{array}{l}\text { Atuação } \\
\text { fisioterapêutica com } \\
\text { ultrassom em } \\
\text { pacientes com } \\
\text { neuropatia } \\
\text { periférica diabética. }\end{array}$ & 2015. & $\begin{array}{l}\text { - Tratamento realizado em } 12 \text { dias } \\
\text { consecutivos. No primeiro dia foi feita a } \\
\text { avaliação, com a utilização de questionário, } \\
\text { em seguida, durante } 10 \text { dias, foi aplicado por } \\
20 \text { minutos o ultrassom e no último dia foi } \\
\text { feita a reavaliação e comparação das } \\
\text { informações. } \\
\text { - Foi usado no estudo o ultrassom da marca } \\
\text { KDL - AVATAR III, de frequência } 1 \mathrm{MHz} \text {, } \\
\text { pulsado com intensidade } 0,5 \mathrm{~W} / \mathrm{cm} 2 \text {. A } \\
\text { aplicação de ultrassom ocorreu da seguinte } \\
\text { forma: } 5 \text { minutos na região plantar e } 5 \text { na } \\
\text { região dorsal dos dois pés, totalizando } 20 \\
\text { minutos diários de terapia. }\end{array}$ \\
\hline $\begin{array}{l}\text { FORTE et } \\
\text { al., }\end{array}$ & $\begin{array}{l}\text { Efeitos do } \\
\text { tratamento } \\
\text { fisioterapêutico na } \\
\text { neuropatia } \\
\text { periférica causada } \\
\text { pelo diabetes: um } \\
\text { relato de caso. }\end{array}$ & 2016. & $\begin{array}{l}\text { - } 24 \text { sessões com duração média de } 50 \\
\text { minutos cada ao longo de } 12 \text { semanas. Foram } \\
\text { realizados exercícios globais e aeróbios, além } \\
\text { de alongamentos da cadeia anterior e } \\
\text { posterior e liberação da fáscia plantar. }\end{array}$ \\
\hline
\end{tabular}

Fonte: Elaborada pelos autores.

No total foram selecionados inicialmente 24 estudos e destes utilizados 5 para compor o quadro com as estratégias que podem ser utilizadas na reabilitação fisioterapêutica em acometidos pela neuropatia diabética.

Dentre as fontes utilizadas para composição dos resultados, 3 são artigos (GOMES et al., 2007; BARROS et al., 2012; YOOSEFINEJAD et al., 2014), uma monografia de Trabalho de Conclusão de Curso (TCC) (FURIERI, 2015) e um 1 trabalho publicado em anais (FORTE et al., 2016). 4 destes foram realizados no Brasil (GOMES et al., 2007; BARROS et al., 2012; FURIERI, 2015; FORTE et al., 2016) e apenas 1 fora do país (YOOSEFINEJAD et al., 2014). 
Todos os estudos, a partir das modalidades de tratamentos fisioterapêutico utilizados, obtiveram resultados positivos, pois todos conseguiram oferecer benefícios para a pessoa acometida pela neuropatia diabética. Isto demonstra o quão importante é a realização de ações terapêuticas visando diminuir os agravos causados por esse mal.

\section{DISCUSSÃO}

A neuropatia diabética (ND) é definida como o dano nervoso periférico, somático ou autonômico, atribuível apenas ao Diabetes Mellitus. Considerando o dano motor, sensorial e funcional causado por esse distúrbio, a atuação da fisioterapia torna-se fundamental para promover um melhor tratamento para os pacientes, contribuindo de forma considerável para a reabilitação das alterações físicas e sensoriais, além da prevenção por meio de exercícios de alongamento, fortalecimento, treino na marcha, equilíbrio e adequação de próteses e órteses para diminuição de sequelas do pé, promovendo uma melhor qualidade de vida (SACCO et al., 2007; SOUSA et al., 2018).

Torna-se importante realizar atividades terapêuticas porque determinado estudo afirma que a atrofia muscular em pacientes neuropáticos, em particular nos músculos intrínsecos do pé, pode causar algumas deformidades e redução dos movimentos do pé e tornozelo, colaborando assim, para o crescimento da pressão plantar. Explica ainda que os movimentos mais prejudicados são a flexão, inversão e eversão do tornozelo e os movimentos da primeira articulação metatarsofalangeana. Esses movimentos quando passam a ser atingidos e insuficientes, diminuem a capacidade do pé de dissolver o choque e as rotações transversais durante a marcha, contribuindo para uma possível patogênese de ulceração plantar (SACCO et al., 2007).

As intervenções fisioterapêuticas surtem efeitos satisfatórios, uma vez que proporcionam uma melhora no fluxo sanguíneo para os membros inferiores de modo a contribuir na cicatrização de ferimentos, diminuição da hipóxia endoneural e no progresso de condução nervosa (BARROS et al., 2012). 
Em concordância, estudo realizado aponta que os exercícios fisioterapêuticos melhoram a circulação dos MMII. Desta forma, tornando possível reduzir a hipóxia endoneural, auxiliar na comunicação neural e melhorar a circulação dos nervos periféricos, resultando em uma melhor condução nervosa e consequentemente na redução dos sintomas do paciente acometido por ND (ABREU, 2018).

Algumas das atividades podem ser feitas com o uso de plataformas vibratórias, pois em uma pesquisa houve a sua utilização em pacientes com neuropatia periférica. Foram realizados exercícios isométricos de agachamento em uma plataforma, sendo utilizado também exercícios ativos funcionais com faixas elásticas e foi observado que ocorreu uma melhora do equilíbrio e da força muscular dos membros inferiores (PORTES, 2015). Já um estudo de metanálise, a partir das informações analisadas, evidenciou que o equilíbrio em pessoas com ND melhorou com a utilização combinada de exercícios resistidos e de equilíbrios (MARONESI et al., 2016).

Por haver diferentes abordagens de exercícios a pacientes com ND, torna-se necessário uma melhor avaliação desses exercícios para possibilitar melhores resultados no tratamento. $O$ tipo de exercício, intensidade, frequência e duração deve ser prescrito com cuidado. Tendo em vista as complicações da doença, o tratamento deve está de acordo com as demandas metabólicas, motoras e proprioceptivas dos indivíduos (LIMA; JUNQUEIRA, 2016; MARONESI et al., 2016).

Por causa da dificuldade do controle glicêmico, o tratamento da neuropatia é difícil e pouco satisfatório (NASCIMENTO et al., 2015). Além disso, o tratamento pode varias de acordo com a duração da fisioterapia e o protocolo de reabilitação estará sujeito ao estágio da ND, no entanto, independente do tempo de tratamento, os objetivos serão os mesmos e a reabilitação busca devolver a funcionalidade dos MMII, adiando assim, a progressão da ND (ABREU, 2018).

De acordo com os dados expostos, é fundamental que a literatura exponha não só as formas de tratar, mas também de prevenir a neuropatia. Algumas medidas que podem ser utilizadas são o controle metabólico do diabetes, pois é, o principal de meio de prevenir a ND. É importante ainda não utilizar em exagero bebidas alcoólicas e tabaco (DELI et al., 2014) (SOCIEDADE BRASILEIRA DE DIABETES, 2014). 
A mensuração regular da glicose também é um instrumento importante para a prevenção das complicações do diabetes. Um artigo realizado constatou que a melhora do controle glicêmico diminui o risco e a progressão das complicações microvasculares e neuropáticas, caracterizando-se como um recurso importante para a prevenção de úlceras e amputações (BARROS et al., 2012).

Portanto, é importante que estudos futuros pesquisem novas ações fisioterapêuticas, pois como visto nessa revisão, ainda são poucas formas de tratamento disponíveis na literatura. Mas além disso, informem sobre medidas profiláticas, para que desta forma, seja possível diminuir os números de casos e os seus possíveis prejuízos.

\section{CONCLUSÃO}

Conclui-se, a partir das informações disponibilizadas nesse estudo, que a fisioterapia contribui para uma redução significativa dos danos causados pela neuropatia diabética. Todavia, além do tratamento, o fisioterapeuta deve agir também na prevenção da DM de modo a evitar complicações, óbitos e custos dessa enfermidade.

O fisioterapeuta precisa ainda ser capaz de identificar qual o tipo de neuropatia diabética que acomete o paciente, pois como visto existem diferentes formas desse agravo se manifestar. Isso é de fundamental importância para que o profissional encontre a forma de tratamento que melhor se adeque as peculiaridades do indivíduo.

Por último, é preciso ressaltar que os dados disponibilizados na literatura são escassos. Isso é algo que precisa ser mudado. É necessário que novos estudos apresentem mais maneiras de tratar a ND, resultando assim, em diminuição dos seus danos.

\section{REFERÊNCIAS}

ABREU, C. O. Modalidades fisioterapêuticas utilizadas em pacientes portadores de neuropatia diabética. 2018. Monografia (Graduação em Fisioterapia) - Faculdade de Educação e Meio Ambiente - FAEMA, Ariquemes, 2018. 
ARAUJO FILHO, A. C. A.; ALMEIDA, P. D.; ARAUJO, A. K. L.; SALES, I. M. M.; ARAÚJO, T. M. E.; ROCHA, S. S. Perfil epidemiológico do Diabetes Mellitus em um estado do nordeste brasileiro. Rev. Fund. Care. Online, [s.l.], v. 9, n. 3, p. 641-647, jul./set. 2017. Disponível em: https://doi.org/10.9789/2175-5361.2017.v9i3.641-647.

BARROS, M. F. A.; MENDES, J. C.; NASCIMENTO, J. A.; CARVALHO, A. G. C. Impacto de intervenção fisioterapêutica na prevenção do pé diabético. Fisioter. Mov. [s.1.], v. 25, n. 4, p. 747-757, out./dez. 2012. Disponível em: https://doi.org/10.1590/S0103-51502012000400007.

BORGES, F. S.; CARDOSO, H. S. G. Avaliação sensório-motora do tornozelo e pé entre idosos diabéticos e não diabéticos. Revista Brasileira de Geriatria e Gerontologia, [s.1.], v. 13, n. 1, p. 93-102, 2010. Disponível em: https://www.scielo.br/j/rbgg/a/F5rpmqWtZHJ73VQBwqGwCHh/?lang=pt\&format=pdf.

DELI, G.; BOSNYAK, E.; PUSCH, G.; KOMOLY, S.; FEHER, G. Diabetic Neuropathies: Diagnosis and Management. Neuroendocrinology, [s.1.], v. 98, p. 267-280, 2014. Disponível em: https://doi.org/10.1159/000358728.

FORTE, J. F.; TAVARES, F. J. R.; MARTINS, C. A.; BARROS, K. V. C.; MONTALVERNE, D. G. B. Efeitos do tratamento fisioterapêutico na neuropatia periférica causada pelo diabetes: um relato de caso. In: XXV Encontro de Extensão, 2016, Fortaleza. Anais [...]. Universidade Federal do Ceará (UFC), 2016.

FURIERI, F. P. M. Atuação fisioterapêutica com ultrassom em pacientes com neuropatia periférica diabética. 2015. Monografia (Graduação em Fisioterapia) - Faculdade de Educação e Meio Ambiente, Ariquemes, 2015.

GALVÃO, T. F.; PEREIRA, M. G. Revisões sistemáticas da literatura: passos para sua elaboração. Epidemiol. Serv. Saúde, [s.1.], v. 23, n. 1, p. 183-184, jan./mar. 2014. Disponível em: http://scielo.iec.gov.br/scielo.php?script=sci_arttext\&pid=S1679-49742014000100018.

GOMES, A. A.; SARTOR, C. D.; JOÃO, S. M. A.; SACCO, I. C. N.; BERNIK, M. M. S. Efeitos da intervenção fisioterapêutica nas respostas sensoriais e funcionais de diabéticos neuropatas. Fisioterapia e Pesquisa, [s.l.], v. 14, n. 1, p. 14-21, 2007. Disponível em: https://www.revistas.usp.br/fpusp/article/view/75500.

INTERNATIONAL DIABETES FEDERATION (IDF). Diabetes atlas. 7. ed. Belgium: International Diabetes Federation, 2015.

LADE, C. G.; MARTINS, J. C. B.; LIMA, L. M.; ALBUQUERQUE, M. R.; TEIXEIRA, R. B.; REIS, J. S.; AMORIM, P. R. S. Nível de atividade física habitual em portadores e não portadores de neuropatia diabética. Revista Brasileira de Atividade Física \& Saúde, [s.1.], v. 21, n. 4, p.324-331, 1 jul. 2016. Disponível em: https://rbafs.org.br/RBAFS/article/view/7635.

LIMA, C. F.; JUNQUEIRA, N. K. B. Efeitos de uma intervenção fisioterapêutica de equilíbrio, propriocepção e coordenação em diabéticos. 2016. Monografia (Graduação em Fisioterapia) - Universidade de Brasília (UNB), Brasília, 2016.

MARONESI, C. T. P.; ZANINI, S. C. C.; OLIVEIRA, L. Z.; BAVARESCO, S. S.; LEGUISAMO, C. P. Exercícios físicos em portadores de neuropatia diabética: revisão sistemática e metanálise de ensaios clínicos randomizados. Fisioter. Pesqui. [s.1.], v. 23, n. 2, p. 216-223, 2016. Disponível em: https://doi.org/10.1590/1809-2950/14649323022016.

MENDES, L. N.; TEIXEIRA, M. S.; MICHELON, C.; BELLO, M. C. Associação entre a periodontite apical e o diabetes mellitus: uma revisão da literatura. Revista da Faculdade de Odontologia - UPF, [s.l.], v. 24, n. 1, p. 58-66, maio 2019. Disponível em: https://doi.org/10.5335/rfo.v24i1.8840.

NASCIMENTO, O. J. M.; PUPE, C. C. B.; CAVALCANTI, E. B. U. Diabetic neuropathy. Rev. Dor. São Paulo, v. 17, p. 46-51, 2016. Disponível em: https://doi.org/10.5935/1806-0013.20160047. 
NASCIMENTO, R. T. L.; LOPES, C. B.; COTTA, D. S.; ALENCAR, N. L. O. R.; VALADÃO, A. F.; MOTTA, P. G. Neuropatia diabética dolorosa - aspectos clínicos, diagnóstico e tratamento: uma revisão de literatura. Revista UNINGÁ, [s.l.], v. 43, p. 71-79, jan./mar. 2015. Disponível em: http://revista.uninga.br/index.php/uninga/article/view/1215.

OLIVEIRA, H. C. C.; FERREIRA, J. L.; COUTINHO, M. L. Importância do enfermeiro na prevenção e cuidado do pé

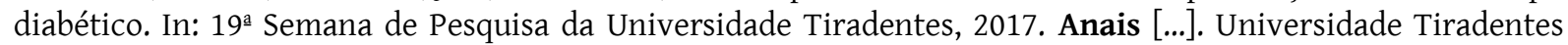
(UNIT), 2017.

PETERMANN, X. B.; MACHADO, I. S.; PIMENTEL, B. N.; MIOLO, S. B.; MARTINS, L. R.; FEDOSSE, E. Epidemiologia e cuidado à Diabetes Mellitus praticado na Atenção Primária à Saúde: uma revisão narrativa. Saúde (Santa Maria), [s.l.], v. 41, n. 1, p. 49-56, jan./jul. 2015. Disponível em: https://periodicos.ufsm.br/revistasaude/article/view/14905/pdf.

PORTES, L. H. Abordagem do fisioterapeuta no diabetes mellitus: revisão de literatura. Arq. Ciênc. Saúde, [s.l.], v. 22, n. 3, p. 9-14, jul./set. 2015. Disponível em: https://doi.org/10.17696/2318-3691.22.3.2015.37.

ROTHER, E. T. Revisão sistemática X revisão narrativa. Acta Paulista de Enfermagem, [s.l.], v. 20, n. 2, abr./jun. 2007. Disponível em: https://doi.org/10.1590/S0103-21002007000200001.

SACCO, I. C. N.; SARTOR, C. D.; GOMES, A. A.; JOÃO, S. M. A.; CRONFLI, R. Avaliação das perdas sensório-motoras do pé e tornozelo decorrentes da neuropatia diabética. Rev. bras. fisioter. [s.l.], v. 11, n. 1, p. 27-33, 2007. Disponível em: https://doi.org/10.1590/S1413-35552007000100006.

SALSABILI, H.; BAHRPEYMA, F.; FOROGH, B.; RAJABALI, S. Dynamic stability training improves standing balance control in neuropathic patients with type 2 diabetes. J. Rehabil. Res. Dev. [s.1.], v. 48, n. 7, p. 775-786, 2011. Disponível em: https://doi.org/10.1682/jrrd.2010.08.0160.

SOCIEDADE BRASILEIRA DE DIABETES. Diretrizes da Sociedade Brasileira de Diabetes 2017-2018. São Paulo: Clannad, 2017.

SOCIEDADE BRASILEIRA DE DIABETES. Diretrizes da Sociedade Brasileira de Diabetes: 2013-2014. São Paulo: AC Farmacêutica, 2014.

SOUSA, J. S.; CONSOLINE, N. A.; ANÍZIO, P. D.; MORSCH, P.; FAGUNDES, D. S. A atuação da fisioterapia na prevenção de úlceras do pé diabético. Revista Científica da Faculdade de Educação e Meio Ambiente, [s.1.], v. 9, n. 1, p. 320-324, jan./jun. 2018. https://doi.org/10.31072/rcf.v9i1.575.

VIEIRA, S.; HOSSN, W. S. Metodologia científica para a área da saúde. 2. ed. Rio de Janeiro: Elsevier, 2015.

YOOSEFINEJAD, A. K.; SHADMEHR, A.; OLYAEI, G.; TALEBIAN, S.; BAGHERI, H. The effectiveness of a single session of Whole-Body Vibration in improving the balance and the strength in type 2 diabetic patients with mild to moderate degree of peripheral neuropathy: a pilot study. J. Bodyw. Mov. Ther. [s.l.], v. 18, n. 1, p. 82-86, 2014. Disponível em: https://doi.org/10.1016/j.jbmt.2013.10.007.

(cc) Br

Este trabalho está licenciado com uma Licença Creative Commons - Atribuição 4.0 Internacional. 